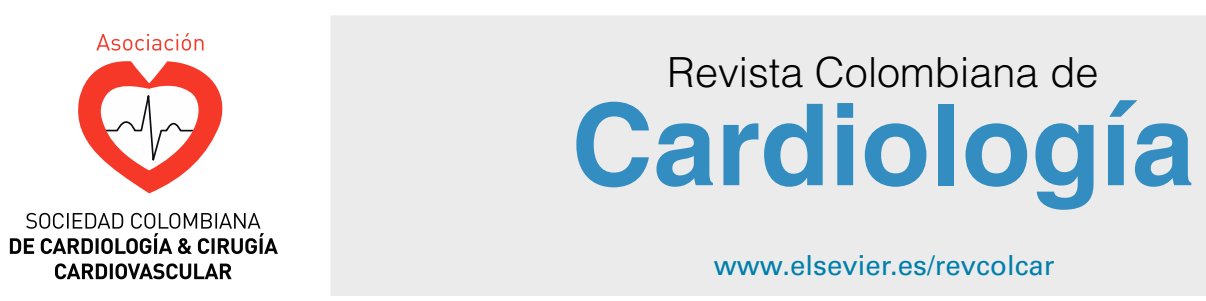

CARDIOLOGÍA DEL ADULTO - ARTÍCULO ORIGINAL

\title{
Utilización de fármacos antihipertensivos, efectividad e inercia clínica en pacientes
}

\author{
Juan Daniel Castrillón-Spitia ${ }^{\mathrm{a}, \mathrm{b}}$, Adriana Franco-Hurtadoc ${ }^{c}$, \\ Carolina Garrido-Hernández ${ }^{c}$, Juliana Jaramillo-Patiñoc, \\ María Alejandra Londoño-Moncada ${ }^{\mathrm{c}}$ y Jorge Enrique Machado-Alba ${ }^{\mathrm{b}, *}$
}

\footnotetext{
a Biomedicina, Fundación Universitaria Autónoma de las Américas, Pereira, Risaralda, Colombia

b Grupo de Investigación en Farmacología y Farmacovigilancia, Universidad Tecnológica de Pereira-Audifarma, S.A., Pereira, Colombia

c Fundación Universitaria Autónoma de las Américas, Semillero de Investigación Médica para la Ciencia y la Comunidad - SIMCC línea de Investigación en Farmacoepidemiologia y Farmacovigilancia. Fundación Universitaria Autónoma de las Américas, Universidad Tecnológica de Pereira, Pereira, Colombia
}

Recibido el 8 de marzo de 2017; aceptado el 18 de agosto de 2017

Disponible en Internet el 17 de abril de 2018

\section{PALABRAS CLAVE Hipertensión arterial; Tratamiento médico óptimo; Atención primaria; Farmacología}

\begin{abstract}
Resumen
Introducción: la hipertensión arterial es una de las principales causas de enfermedad cardiovascular. Se encuentra una limitada caracterización local de su tratamiento en nuestra población. Metodología: estudio de corte transversal sobre la prescripción de fármacos antihipertensivos, la efectividad del tratamiento y la inercia clínica en pacientes atendidos en 2014. Se estableció la efectividad según el Sevent Report of the Joint National Committee y se definió inercia clínica como la falta de modificaciones para alcanzar la meta terapéutica. Se incluyeron variables sociodemográficas y farmacológicas. Se aplicaron modelos de regresión logística.

Resultados: de un total de 4.195 pacientes, se obtuvo una muestra aleatoria de 309 , el $54,7 \%$ eran mujeres y $52,8 \%$ tenían más de 60 años. Los antihipertensivos más utilizados fueron losartán e hidroclorotiazida ( $20,8 \%$ cada uno). Se halló un control de cifras tensionales en el $75,3 \%$ de un total de 675 consultas y de las 167 visitas que no presentaron control tensional se evidenció inercia clínica en 47,9\%. Tener prescrito concomitantemente un antidiabético se asoció con menor probabilidad de que se presentara inercia clínica ( $p=0,032 ;$ OR: 0,21; IC $95 \%$ : 0,051. 0,879).
\end{abstract}

\footnotetext{
* Autor para correspondencia.

Correo electrónico: machado@utp.edu.co (J.E. Machado-Alba).
} 
Conclusión: la proporción de pacientes que no logran las metas de control de tensión arterial y la frecuencia de inercia clínica es menor que en otras series de pacientes.

(C) 2018 Sociedad Colombiana de Cardiología y Cirugía Cardiovascular. Publicado por Elsevier España, S.L.U. Este es un artículo Open Access bajo la licencia CC BY-NC-ND (http:// creativecommons.org/licenses/by-nc-nd/4.0/).

\section{KEYWORDS}

Arterial hypertension; Optimal medical treatment; Primary care; Pharmacology

\section{Antihypertensive drug use, efficacy, and clinical inertia in patients}

\begin{abstract}
Introduction: Arterial hypertension is one of the main causes of cardiovascular disease. The characteristics of its treatment in the population at a local level are relatively unknown.

Methods: A cross-sectional study was conducted on the prescribing of antihypertensive drugs, the efficacy of the treatment, as well as the clinical inertia in patients seen in the year 2014 . The efficacy was established according to the Seventh Report of the Joint National Committee, and clinical inertia was defined as lack of modifications to achieve the therapeutic aim. Sociodemographic and pharmacological variables were included in the logistical regression models.

Results: Out of a total sample of 4,195 patients, a randomised sample of 309 patients was obtained, of which $54.7 \%$ were women, and $52.8 \%$ were over 60 years of age. The antihypertensive drugs most used were losartan and hydrochlorothiazide (20.8\% each). Blood pressure control was observed in $75.3 \%$ of a total of 675 clinical visits, and of the 167 visits where the blood pressure was not controlled, there was evidence of clinical inertia in $47.9 \%$. To have a concomitant antidiabetic drug prescribed was associated with a lower possibility of there being clinical inertia ( $P=.032$; OR: $0.21 ; 95 \% \mathrm{cl}$ : 0.051-0.879).

Conclusion: The proportion of patients that do not achieve the targets of blood pressure control, as well as the frequency of clinical inertia, are lower than those found in other patient series. (C) 2018 Sociedad Colombiana de Cardiología y Cirugía Cardiovascular. Published by Elsevier España, S.L.U. This is an open access article under the CC BY-NC-ND license (http:// creativecommons.org/licenses/by-nc-nd/4.0/).
\end{abstract}

\section{Introducción}

La hipertensión arterial (HTA) es la enfermedad crónica más frecuente en la práctica clínica y una de las principales causas de riesgo cardiovascular. Tiene una prevalencia mundial entre $20-45 \%{ }^{1}$ y nacional del $23 \%$ en la población adulta; no obstante, en Estados Unidos y en Colombia tan solo el 34 y el $40 \%$ respectivamente, han logrado el control de las cifras ${ }^{3}$.

La Organización Mundial de la Salud (OMS) menciona que el porcentaje de adherencia al tratamiento de los pacientes con enfermedades crónicas llega a un $50 \%$ en países desarrollados, y que aproximadamente el $20 \%$ de la población mundial padece HTA mientras que sólo entre 3 y $34 \%$ de este grupo controla su presión con un tratamiento antihipertensivo ${ }^{2}$.

El riesgo para su desarrollo aumenta con la edad, estilos de vida y comorbilidades asociadas ${ }^{1,4-6}$. Se han establecido diferentes estrategias preventivas para la HTA, relacionadas específicamente con estilos de vida, en especial la dieta y el ejercicio. Una vez el diagnóstico se ha establecido, se busca limitar el daño en órganos blanco por medio de medicamentos; no obstante, también se ha evidenciado falta de control y mala adherencia al tratamiento farmacológico ${ }^{2}$.

Para el manejo de la HTA se usan los diuréticos, los antagonistas de los canales de calcio (ACC), los inhibidores del sistema renina-angiotensina-aldosterona, los $\beta$-bloqueadores (BB), entre otros, ya sea en monoterapia o combinados, para así obtener las cifras de tensión arterial
(TA) deseadas ${ }^{1,4,5,7,8}$. La inercia clínica se define como la tendencia de los médicos de no modificar el tratamiento a pesar de estar indicado, hecho que se explica principalmente por temor del médico a intensificar la terapia o incapacidad de reconocer la falta de control (desconociendo las guías de práctica clínica, subestimando el riesgo del paciente y por falta de motivación en el control a largo plazo) $)^{9,10}$. Se ha reportado falta de control de la presión arterial en el 18,3\% de las consultas de pacientes de 20 ciudades colombianas, y de estos el $81,8 \%$ presentaron inercia clínica; además, se halló que era más común en aquellos pacientes que usaban antidiabéticos ${ }^{11}$.

Es por esto, que a pesar de que en la literatura se establece el tratamiento farmacológico más adecuado para iniciar, aún se puede evidenciar un control inapropiado de la TA en muchos pacientes ${ }^{7,12}$. Así, se buscó determinar la efectividad del tratamiento y la magnitud de la inercia clínica en una población de pacientes atendidos en un hospital de primer nivel y establecer los factores que influencian el control de la HTA.

\section{Materiales y métodos}

Estudio descriptivo, de corte transversal, que incluye individuos con HTA, mayores de 18 años, de cualquier sexo, atendidos en consulta médica para control de esta morbilidad entre el $1^{\circ}$. de enero y el 31 de diciembre de 2014 
en el Hospital San Pedro y San Pablo del municipio de La Virginia, Risaralda, Colombia.

Los criterios de exclusión solo se tuvieron en cuenta para aquellos pacientes menores de 18 años y los que no asistían a los controles establecidos por el programa del hospital.

A partir de una población de 4.195 pacientes con HTA, se realizó un muestreo aleatorio [proporción esperada de control de $40 \%{ }^{13}$, error del $5,0 \%$, intervalo de confianza (IC): 95\%]; para la toma de la muestra se usaron reportes locales de control de presión arterial ya que la población de estudio tiene características propias y se requerían datos de una población de características similares para una inferencia estadística correcta ${ }^{13}$. La información la obtuvieron estudiantes de Medicina a partir de todas las historias clínicas de las consultas para control de la presión arterial durante el periodo de estudio, previo consentimiento por parte del hospital, y luego fue revisada por uno de los investigadores; se obtuvieron las siguientes variables:

1. Sociodemográficas: edad (años), sexo (hombre/mujer), estado civil (casado/separado/ soltero/unión libre/viudo).

2. Medidas antropométricas y paraclínicos (primera consulta de control): índice de masa corporal [IMC $\left(\mathrm{kg} / \mathrm{m}^{2}\right)$, creatinina $(\mathrm{mg} / \mathrm{dl})$, glicemia $(\mathrm{mg} / \mathrm{dl})$, colesterol total $(\mathrm{mg} / \mathrm{dl})$, lipoproteínas de baja densidad (LDL) $(\mathrm{mg} / \mathrm{dl})$, lipoproteínas de alta densidad $(\mathrm{HDL})(\mathrm{mg} / \mathrm{dl})$, triglicéri$\operatorname{dos}(\mathrm{mg} / \mathrm{dl})]$.

3. Comorbilidad/factores de riesgo: diabetes mellitus tipo 2 (si/no), tabaquismo (si/no), dislipidemia (si/no), antecedentes personales de enfermedad coronaria (si/no), Antecedentes cardiacos (si/no), infarto agudo de miocardio (IAM) (si/no), accidente cerebrovascular (ACV) (si/no), enfermedad renal crónica (ERC) (si/no), sedentarismo (si/no), obesidad (si/no), otros antecedentes (si/no), ¿cuáles? (nombre de otros antecedentes).

4. Medicamentos antihipertensivos: nombre del medicamento, dosis, cantidad entregada en todas las consultas. Se utilizó la dosis diaria definida (DDD) como unidad de medida de la utilización de los siguientes fármacos: inhibidores de la enzima convertidora de angiotensina (iECA), antagonistas del receptor de angiotensina-2 (ARA-II), diuréticos, BB y ACC.

5. Comedicación dispensada: a) antiplaquetarios; b) antiinflamatorios no esteroideos (AINE); c) antidiabéticos, d) hipolipemiantes e) antiulcerosos.

6. Seguimiento y efectividad: reporte de medición de la presión arterial sistólica y diastólica en las consultas durante el año de estudio, efectividad de la terapia en cada consulta (si/no) y proporción de consultas en metas de control y consejería sobre cambios del estilo de vida. La efectividad fue establecida con base en las recomendaciones del Sevent Report of the Joint National Committee - 7JNC ${ }^{14}$ (se aceptó como efectividad a las cifras tensionales en metas de control para la edad en cada consulta de los pacientes en el periodo de estudio, con base en las recomendaciones del $7 \mathrm{JNC}$ ).

7. Inercia clínica (si/no): se definió como la falta de modificaciones por parte del médico (adición de antihipertensivo, aumento de la dosis, cambio de medicamentos, recomendaciones de cambios de estilos de vida) para alcanzar la meta terapéutica establecida en al menos una consulta médica.

No se tuvo en cuenta como variable el tiempo de evolución de la HTA en la población de estudio, ya que el diseño del mismo no permitía identificar la antigüedad de la HTA en los pacientes.

Este trabajo recibió el aval del comité de Bioética de la Universidad Tecnológica de Pereira, en la categoría de investigación sin riesgo y se respetaron los principios de confidencialidad dados por la Declaración de Helsinki.

La muestra del estudio se obtuvo a través de EPIDAT 3.1. Los datos se analizaron con el paquete estadístico SPSS Statistics versión 22.0 para Windows (IBM. EE.UU). Se establecieron frecuencias y proporciones; se utilizaron pruebas t de Student o ANOVA para la comparación de variables cuantitativas y $X^{2}$ para categóricas. Se aplicaron modelos de regresión logística binaria usando como variable dependiente la presencia o no de inercia clínica en la terapia antihipertensiva y como variables independientes aquellas que se asociaron de manera estadísticamente significativa en los análisis bivariados. Se determinó como nivel de significancia estadística una $p<0,05$.

\section{Resultados}

Se obtuvo una muestra aleatoria de 309 pacientes. Las variables clínicas y sociodemográficas se muestran en la tabla 1. Hubo un ligero predominio de mujeres $(54,7 \%)$ y de mayores de 60 años $(52,8 \%)$ con una media de edad de 59,9 $\pm 13,6$ años.

Los factores de riesgo más frecuentes fueron dislipidemia, sedentarismo y obesidad; las comedicaciones más empleadas fueron los hipolipemiantes y los antiagregantes plaquetarios, mientras que los antihipertensivos más utilizados fueron losartán e hidroclorotiazida (tabla 2). La media de antihipertensivos usados por paciente fue de 2,55 $\pm 1,08$.

La mitad de los pacientes en quienes se usó espironolactona no presentaba falla cardiaca asociada (9 casos), un paciente la utilizaba en monoterapia y dos como segunda línea $(5,5 \%$ y $11,1 \%$ respectivamente); el $10,3 \%$ de los pacientes en los que se usó prazosín lo hizo de primera $(4,1 \%)$ o segunda línea $(6,2 \%)$. En aquellos que recibían furosemida el $11,5 \%(n=7)$ los usó como segunda línea de manejo, de los cuales solo tres presentaba falla cardiaca asociada.

Se halló un control de cifras tensionales en el 75,3\% $(n=508)$ de un total de 675 consultas. De las restantes 167 visitas sin control tensional la inercia clínica estuvo presente en 80 de estas $(47,9 \%)$ (tabla 3$)$.

Un total de 6 de 45 pacientes a quienes se les había agregado un nuevo antihipertensivo en la primera consulta, tuvieron inercia clínica en alguna de las siguientes consultas del año. Además, 47 (18,6\%) pacientes que estaban controlados en la segunda consulta, tuvieron inercia clínica en algunas de las siguientes consultas.

\section{Análisis multivariado}

Al realizar la regresión logística de la presencia de inercia clínica y las variables que se encontraron asociadas en el análisis bivariado, se halló que haber adicionado un nuevo 
Tabla 1 Características sociodemográficas y clínicas de 309 pacientes con diagnóstico de hipertensión arterial que asistieron a control en el Hospital San Pedro y San Pablo de La Virginia, Risaralda, 2014

\begin{tabular}{|c|c|c|}
\hline Variables & Número & Porcentaje \\
\hline \multicolumn{3}{|l|}{ Sociodemográficas } \\
\hline $\begin{array}{l}\text { Estado civil (casado/separado/ } \\
\text { soltero/unión libre/viudo) }\end{array}$ & $68 / 19 / 142 / 56 / 24$ & $22 / 6,1 / 46 / 18,1 / 7,8$ \\
\hline Edad (>60 años/<60 años) & $163 / 146$ & $52,8 / 47,2$ \\
\hline Sexo (hombre/mujer) & $140 / 169$ & $45,3 / 54,7$ \\
\hline \multicolumn{3}{|l|}{ Variables clínicas } \\
\hline Glicemia $(<100 />100) \mathrm{mg} / \mathrm{dl}$ & $164 / 88$ & $53,1 / 28,5$ \\
\hline Colesterol total $(<200 />200) \mathrm{mg} / \mathrm{dl}$ & $136 / 107$ & $44,0 / 34,6$ \\
\hline $\operatorname{IMC}^{\mathrm{a}}(<25 />25) \mathrm{kg} / \mathrm{m}^{2}$ & $92 / 217$ & $29,8 / 70,2$ \\
\hline $\mathrm{LDL}^{\mathrm{b}}(<70 />70) \mathrm{mg} / \mathrm{dl}$ & $52 / 188$ & $16,8 / 60,8$ \\
\hline $\mathrm{HDL}^{\mathrm{c}}(<30 />30) \mathrm{mg} / \mathrm{dl}$ & $8 / 231$ & $2,6 / 74,8$ \\
\hline Triglicéridos $(<200 />200) \mathrm{mg} / \mathrm{dl}$ & $156 / 86$ & $50,5 / 27,8$ \\
\hline \multicolumn{3}{|l|}{ Comorbilidades } \\
\hline Dislipidemia & 237 & 76,7 \\
\hline Sedentarismo & 225 & 72,8 \\
\hline Obesidad & 217 & 70,2 \\
\hline Diabetes mellitus & 82 & 26,5 \\
\hline Tabaquismo & 54 & 17,5 \\
\hline Antecedentes cardiacos & 43 & 13,9 \\
\hline Antecedentes infarto agudo de miocardio & 11 & 3,6 \\
\hline Antecedente enfermedad cerebrovascuar & 13 & 4,2 \\
\hline Enfermedad renal crónica & 9 & 2,9 \\
\hline $\begin{array}{l}\text { Otros (polineuropatía diabética, nefropatía, } \\
\text { retinopatía, hiperglicemia aislada } \\
\text { hipotiroidismo, angiopatía periférica, } \\
\text { insuficiencia cardiaca congestiva, vasculitis). }\end{array}$ & 59 & 19,1 \\
\hline \multicolumn{3}{|l|}{ Comedicaciones: } \\
\hline Hipolipemiantes (uno/dos) & $241 / 62$ & $78,0 / 20,0$ \\
\hline Anti-agregantes plaquetarios & 220 & 71,2 \\
\hline Antiulcerosos & 123 & 39,8 \\
\hline Antidiabéticos (uno/dos) & $41 / 69$ & $13,3 / 22,3$ \\
\hline Antinflamatorios no esteroideos & 33 & 10,7 \\
\hline Insulina & 26 & 8,4 \\
\hline \multicolumn{3}{|l|}{ Inercias clínicas por consultas ( $n=167)$} \\
\hline $\begin{array}{l}\text { Número de pacientes con inercia en } 1 \\
\text { consulta }\end{array}$ & 53 & 31,7 \\
\hline $\begin{array}{l}\text { Número de pacientes con inercia en } 2 \\
\text { consulta }\end{array}$ & 23 & 13,8 \\
\hline $\begin{array}{l}\text { Número de pacientes con inercia en } 3 \\
\text { consulta }\end{array}$ & 4 & 2,4 \\
\hline Número de pacientes con alguna inercia & 80 & 47,9 \\
\hline \multicolumn{3}{|l|}{ Control de cifras por consulta } \\
\hline $\begin{array}{l}\text { Número de pacientes con control en } 1 \\
\text { consulta }\end{array}$ & $211 / 309$ & 68,3 \\
\hline $\begin{array}{l}\text { Número de pacientes con control en } 2 \\
\text { consulta }\end{array}$ & $253 / 309$ & 81,9 \\
\hline $\begin{array}{l}\text { Número de pacientes con control en } 3 \\
\text { consulta }\end{array}$ & $40 / 50$ & 80,0 \\
\hline $\begin{array}{l}\text { Número de pacientes con control en } 4 \\
\text { consulta }\end{array}$ & $4 / 7$ & 57,1 \\
\hline
\end{tabular}

a (Índice de masa corporal),

b (Low density lipoprotein),

c (High density lipoprotein). 
Tabla 2 Antihipertensivos usados en el manejo de la hipertensión arterial en el Hospital San Pedro y San Pablo de La Virginia, Risaralda, 2014

\begin{tabular}{|c|c|c|c|}
\hline Antihipertensivo & $\mathrm{nDDD}^{\mathrm{a}}$ & Frecuencia & Porcentaje \\
\hline Losartá n & 2,17 & 165 & 20,8 \\
\hline Hidroclorotiazida & 0,80 & 165 & 20,8 \\
\hline Amlodipino & 2,03 & 99 & 12,5 \\
\hline Enalapril & 3,28 & 99 & 12,5 \\
\hline Metoprolol tartrato & 1,12 & 79 & 10,0 \\
\hline Furosemida & 1,41 & 61 & 7,7 \\
\hline Prazosín & 0,39 & 48 & 6,0 \\
\hline Verapamilo & 1,01 & 21 & 2,6 \\
\hline Espironolactona & 0,38 & 18 & 2,3 \\
\hline Nifedipino & 2,06 & 16 & 2,0 \\
\hline Carvedilol & 0,68 & 9 & 1,1 \\
\hline Captopril & 2,50 & 5 & 0,6 \\
\hline Propranolol & 0,62 & 4 & 0,5 \\
\hline Nimodipino & 0,16 & 4 & 0,5 \\
\hline \multicolumn{4}{|c|}{ Combinaciones más frecuentes } \\
\hline \multicolumn{2}{|c|}{ Losartán/hidroclorotiazida } & 59 & 19,3 \\
\hline \multicolumn{2}{|c|}{ Enalapril/Hidroclorotiazida } & 46 & 14,9 \\
\hline \multicolumn{2}{|l|}{ Amlodipino/Losartán } & 36 & 11,7 \\
\hline \multicolumn{2}{|l|}{ Metoprolol/Losartán } & 16 & 5,2 \\
\hline \multicolumn{2}{|c|}{ Metoprolol/Hidroclorotiazida } & 12 & 2,9 \\
\hline \multicolumn{2}{|c|}{ Captopril/Hidroclorotiazida } & 3 & 0,9 \\
\hline
\end{tabular}

${ }^{a}$ (relación entre la dosis media y la dosis diaria definida).

antihipertensivo en la primera consulta y tener control de las cifras de presión arterial en la primera y segunda consulta se asociaron como factor de riesgo para que existiera inercia clínica en la población durante una de las siguientes consultas de control del año. También se encontró que los pacientes que tenían prescrito un antidiabético de manera concomitante, se asociaron como un factor protector para la inercia clínica para una de las siguientes consultas del año. En la tabla 4 se exponen las variables asociadas en el análisis multivariado.

\section{Discusión}

El estudio logró identificar la prescripción de fármacos antihipertensivos, la efectividad de la terapia y la inercia clínica en un grupo de pacientes de La Virginia, Risaralda en el año 2014. El antihipertensivo más usado fue losartán seguido de hidroclorotiazida ${ }^{6,14-17}$; no obstante, se ha encontrado que el inicio de cualquiera de los cuatro grupos farmacológicos recomendados por las guías de práctica clínica tiene reducciones similares en las cifras de la presión arterial cuando se emplean como monoterapia ${ }^{15}$. Losartán en conjunto con hidroclorotiazida $(n=59 ; 19,28 \%)$ fue la combinación más usada en estos pacientes, dato relevante puesto que se ha demostrado que el control de las cifras de tensión puede ser más efectivo con la combinación de estos, por lo que es recomendado como primera línea de manejo por el $7 J_{N C^{14,18}}$; sin embargo la combinación benazepril y amlodipino frente a la combinación benazepril más hidroclorotiazida ha mostrado beneficios en pacientes con hipertensión en estadio 2, con reportes de hasta un $20 \%$ en la reducción del riesgo cardiovascular, siendo recomendados por la Sociedades Americana
Tabla 3 Ajuste y cambios de la terapia antihipertensiva en el manejo de la hipertensión arterial en el Hospital San Pedro y San Pablo de La Virginia, Risaralda, 2014

\begin{tabular}{|c|c|c|}
\hline Control & Frecuencia & Porcentaje \\
\hline \multicolumn{3}{|l|}{$\begin{array}{l}\text { Control primera } \\
\text { consulta }(n=309)\end{array}$} \\
\hline Ajuste de terapia & 53 & 17,2 \\
\hline Aumento de dosis & 18 & 5,8 \\
\hline Nuevo anti-HTA ${ }^{a}$ & 45 & 14,6 \\
\hline Cambio de terapia & 50 & 16,2 \\
\hline \multicolumn{3}{|c|}{$\begin{array}{l}\text { Control segunda consulta } \\
(n=309)\end{array}$} \\
\hline Ajuste de terapia & 109 & 35,3 \\
\hline Aumento de dosis & 33 & 10,7 \\
\hline Nuevo anti-HTA (2) & 94 & 30,4 \\
\hline Cambio de terapia & 65 & 21,0 \\
\hline \multicolumn{3}{|c|}{$\begin{array}{l}\text { Control tercera consulta } \\
(n=50)\end{array}$} \\
\hline Ajuste de terapia & 16 & 5,2 \\
\hline Aumento de dosis & 6 & 1,9 \\
\hline Nuevo anti-HTA (3) & 13 & 4,2 \\
\hline Cambio de terapia & 11 & 3,6 \\
\hline \multicolumn{3}{|l|}{$\begin{array}{l}\text { Control cuarta consulta } \\
(n=7)\end{array}$} \\
\hline Ajuste de terapia & 3 & 1,0 \\
\hline Aumento de dosis & 1 & 0,3 \\
\hline Nuevo anti-HTA (4) & 3 & 1,0 \\
\hline Cambio de terapia & 2 & 0,6 \\
\hline \multicolumn{3}{|c|}{ Nuevos antihipertensivos } \\
\hline \multicolumn{3}{|c|}{$\begin{array}{l}\text { Nuevo anti-HTA (primera } \\
\text { consulta) }\end{array}$} \\
\hline Losartán & 10 & 3,2 \\
\hline Hidroclorotiazida & 9 & 2,9 \\
\hline Amlodipino & 6 & 1,9 \\
\hline \multicolumn{3}{|c|}{$\begin{array}{l}\text { Nuevo anti-HTA (segunda } \\
\text { consulta) }\end{array}$} \\
\hline Amlodipino & 19 & 6,1 \\
\hline Losartán & 19 & 6,1 \\
\hline Hidroclorotiazida & 17 & 5,5 \\
\hline Metoprolol tartrato & 14 & 4,5 \\
\hline \multicolumn{3}{|c|}{$\begin{array}{l}\text { Nuevo anti-HTA (tercera } \\
\text { consulta) }\end{array}$} \\
\hline Prazosin & 3 & 1,0 \\
\hline Hidroclorotiazida & 2 & 0,6 \\
\hline Amlodipino & 2 & 0,6 \\
\hline Carvedilol & 2 & 0,6 \\
\hline \multicolumn{3}{|l|}{$\begin{array}{l}\text { Nuevo anti-HTA (cuarta } \\
\text { consulta) }\end{array}$} \\
\hline Furosemida & 1 & 0,3 \\
\hline Metoprolol tartrato & 1 & 0,3 \\
\hline Amlodipino & 1 & 0,3 \\
\hline
\end{tabular}

a (Antihipertensivo). 
Tabla 4 Variables asociadas con la inercia clínica en el manejo de la hipertensión arterial en el Hospital San Pedro y San Pablo de La Virginia, Risaralda, 2014

IC $95 \%$ d

\begin{tabular}{|c|c|c|c|}
\hline Variables & Sig. & $\mathrm{OR}^{\mathrm{c}}$ & Superio \\
\hline Control hipertensión arteriala & $<0,001$ & $5,9963,153$ & 11,402 \\
\hline Obesidad & 0,052 & $1,8560,994$ & 3,468 \\
\hline Diabetes mellitus & 0,260 & $2,3070,538$ & 9,890 \\
\hline Enfermedad renal crónica & 0,595 & $0,6590,142$ & 3,057 \\
\hline Antidiabético & 0,032 & $0,2130,051$ & 0,879 \\
\hline Insulina & 0,857 & $0,8950,269$ & 2,980 \\
\hline Nuevo antihipertensivo & 0,003 & $5,2991,760$ & 15,953 \\
\hline Control hipertensión arterial ${ }^{\mathrm{b}}$ & 0,003 & $2,9361,426$ & 6,047 \\
\hline Constante & 0,004 & 0,298 & \\
\hline
\end{tabular}

a (Primera consulta),

b (Segunda consulta);

c OR: razón de disparidad.

d IC $95 \%$ : intervalo de confianza de $95 \%$.

y Europea de Hipertensión como agentes de primera línea para esta población ${ }^{19-21}$.

Se encontró que tanto losartán como enalapril se usaron a DDD superiores a las recomendadas. Un trabajo realizado por este mismo grupo de investigación mostró que a 6.094 sujetos (14,6\% de una población de dos Empresas Promotoras de Salud de la ciudad de Bogotá a los cuales se le prescribía losartán) se les formuló más de $100 \mathrm{mg}$ /día de losartán, dosis con la cual supera su pico de efectividad, con evidencia limitada que muestre su impacto sobre la morbilidad y mortalidad frente a dosis menores y que aumenta el riesgo de reacciones adversas como la hiperkalemia ${ }^{22}$. Al ser poblaciones diferentes, es preocupante encontrar estos fármacos en dosis superiores a las recomendadas, por lo cual la identificación de dichos errores en la formulación y la realización de intervenciones como las llevadas a cabo por este grupo de investigación o por Fernández et al. ${ }^{22,23}$, que demuestran reducción en las formulaciones inadecuadas, deben seguirse desarrollando.

El concepto de inercia clínica abarca la falla en la iniciación de un tratamiento o la falta de intensificación del mismo a pesar de que las metas no se hayan alcanzado, siendo identificado este parámetro como uno de los principales causantes de la HTA no controlada ${ }^{24}$, que asociado a otras variables como hábitos de vida no saludables y poca adherencia a la terapia farmacológica pueden conducir a peores resultados en los pacientes, y por ende a incremento de la mortalidad por enfermedad coronaria y por $\mathrm{ACV}^{24}$. En este estudio se presentó inercia en $47,9 \%$ de las consultas, estableciéndose una asociación con diferentes variables, en contraste con una investigación realizada en 20 ciudades de Colombia en la que se encontró que el $81,8 \%$ de las consultas que no lograron el control de la tensión arterial, presentaban inercia clínica ${ }^{11}$; por otra parte, una investigación realizada en España mostró que el 70,9\% de los pacientes tenían pobre control de las cifras de tensión y que al $42,1 \%$ no se le realizó ningún tipo de modificación en la terapia a pesar de no estar en metas ${ }^{25}$. La menor frecuencia de inercia clínica encontrada en el presente estudio puede relacionarse con que en la actualidad el hospital de La Virginia es piloto en la aplicación de las recomendaciones trazadoras del Ministerio de Salud en HTA y diabetes mellitus, y que existen médicos dedicados a su aplicación.

También, en China y Estados Unidos se ha reportado una inercia clínica del $31 \%$ y $16,8 \%$ respectivamente (IC95\%: $15,8 \%$ a 17,9\% en los Estados Unidos); en el estudio chino se describe que el $70 \%$ de los médicos y el $85 \%$ de los pacientes creían que se alcanzaron los objetivos ${ }^{26,27}$.

Por otra parte, en un estudio realizado en Pereira, se determinó que sólo al $58,2 \%$ de la población estudiada se le realizaron modificaciones en el tratamiento antihipertensivo ${ }^{28}$, lo que indica que el $50 \%$ son factores asociados al prescriptor como las fallas para valorar las metas del tratamiento, no fijar metas claras de presión arterial, subestimar las necesidades de los pacientes y no identificar o manejar las comorbilidades asociadas, mientras que un $30 \%$ se asoció a factores relacionados con el paciente, como los hábitos de vida no saludables y la pobre adherencia farmacológica, y solo un $20 \%$ a factores del sistema sanitario como la carencia de guías de práctica clínica ${ }^{28}$.

La frecuencia de uso de metoprolol tartrato debe ser objeto de mayor discusión debido a que su efectividad puede ser menor que la de otros $\mathrm{BB}^{29}$. Así mismo, se identificó un uso inadecuado de la espironolactona en el 16,6\% de los pacientes, puesto que a pesar de tener efectos beneficiosos en insuficiencia cardiaca congestiva (ICC), no se ha probado su efectividad en HTA y se recomienda emplearse como manejo de tercera o cuarta línea ${ }^{1}$. Pese a que el uso de los $\alpha$-bloqueadores en nuestro estudio es frecuente, no se recomiendan para iniciar la terapia (10,3\% de los casos), visto que la evidencia científica ha demostrado que aumenta la tasa de eventos cerebrovasculares (y empeora el control de la ICC al equipararse con el uso de un diurético tipo tiazida) ${ }^{1,2,5,6,8,13,30}$.

Se encontró una asociación entre la formulación de un antidiabético y no presentar inercia clínica en el manejo de la HTA, dato que es relevante. En pacientes con diabetes y ERC, el objetivo del tratamiento antihipertensivo es triple: reducir la presión arterial, minimizar el riesgo de complicaciones cardiovasculares y retardar la progresión de la morbilidad. En España, el estudio Presión Arterial en la Población Española en los Centros de Atención Primaria (PRESCAP) 2010, en donde el 31\% de sujetos presentaba concomitantemente diabetes, mostró que el $50 \%$ tenía un control adecuado de la $\mathrm{TA}^{31}$. Sin embargo, una revisión sistemática estimó que el buen control tensional en pacientes con diabetes e HTA es de tan solo el $12 \%{ }^{32}$. Conocer el control de la TA y su asociación con el manejo farmacológico en pacientes con diabetes o ERC, es un punto interesante a tener en cuenta para el desarrollo de futuros trabajos de investigación.

Entre las limitaciones del estudio se debe tener en cuenta que las cifras de TA fueron tomadas de los registros encontrados en las historias clínicas y no a cada sujeto con un mismo equipo estandarizado; no obstante, el hospital cuenta con el servicio de calibración de los equipos una vez cada tres meses y además las consultas de control elegidas en el estudio fueron atendidas por médicos generales que tienen la experticia y la calificación para realizar dicho procedimiento. Tampoco se conocen las razones de la elección de los antihipertensivos formulados por parte de los clínicos o en aquellos casos en los que no se usó, modificó o cambió 
un antihipertensivo. La población objeto de estudio fueron adultos de un hospital de primer nivel de un municipio y en su mayoría pertenecientes al régimen subsidiado del Sistema General de Seguridad Social en Salud (SGSSS), por lo que la información de este estudio se puede extrapolar solo a poblaciones con características similares.

López-Simarro ${ }^{33}$ ha descrito recomendaciones para reducir la inercia clínica, que pueden ser aplicables en el control de las diferentes patologías crónicas. Se describen determinantes del profesional, del paciente y del sistema de salud que afectan el acto médico y llevan a inercia clínica; recomiendan estrategias como la educación médica continua, el estudio de guías de práctica clínica basadas en la evidencia, la enseñanza del concepto de la inercia clínica y su impacto en la salud de los pacientes, los incentivos económicos a los profesionales, lo optimización de la relación médico-paciente, involucrar al paciente en el manejo (empleo de mensajes cortos y de fácil lectura que informen a los pacientes sobre los objetivos establecidos y les alienten a comentar su tratamiento con el médico), además de la utilización de auditorías y retorno de la información a los médicos, con intervenciones educativas a estos últimos ${ }^{21,22}$. También describen recordatorios informáticos en la historia clínica sobre la ausencia de cumplimientos de metas, reacciones adversas o tratamientos más efectivos, entre otras intervenciones $^{33,34}$.

Deberían adelantarse nuevas investigaciones acerca de la prescripción de antihipertensivos en otras instituciones, para evaluar la adherencia a las guías y el impacto de dicho tratamiento tanto en la salud de los pacientes de nuestra localidad como en el SGSSS.

\section{Conclusiones}

Los pacientes del Hospital San Pedro y San Pablo de La Virginia, están siendo tratados con medicamentos de alto valor terapéutico, propuestos en su mayoría por las guías de práctica clínica, a dosis adecuadas o superiores a las recomendadas. Además, se halló una efectividad comparativamente alta en el control de la HTA y una inercia clínica menor que puede ser la explicación del éxito logrado por el programa de atención establecido por dicha institución.

La información debe ser utilizada para establecer estrategias de mejoramiento de los programas de atención primaria enfocadas a evitar que se presente inercia clínica, lo que seguramente impactará en menores complicaciones de estos pacientes.

\section{Financiación}

Universidad Tecnológica de Pereira y Audifarma S.A.

\section{Conflicto de intereses}

Los autores declaran no tener ningún conflicto de intereses.

\section{Bibliografía}

1. Grupo de Trabajo de la SEC., sobre la guía de hipertensión arterial, ESC/ESH., 2013, revisores expertos de la guía de hipertensión arterial, ESC/ESH., 2013, Comité de Guías de Práctica Clínica de la SEC. Comments on the ESC/ESH guidelines for the management of arterial hypertension 2013. A report of the Task Force of the Clinical Practice Guidelines Committee of the Spanish Society of Cardiology. Rev Esp Cardiol (Engl Ed). 2013;66:842-7.

2. De Geest S, Sabaté E. Adherence to long-term therapies: evidence for action. Eur J Cardiovasc Nurs. 2003;2:323.

3. Mendivil c. Guías de práctica clínica basadas en la evidencia sobre el tamizaje, diagnóstico y tratamiento de la hipertensión arterial. Universidad nacional de Colombia; 2007 [Acceso abril de 2015). Disponible en: http://www.doctoradiabetes.com. co/guias/13-\%20Guias\%20HTA\%20de\%20Univ\%20Nacional\%2020 07\%20-\%20Castellano.pdf

4. Gudsoorkar PS, Tobe SW. Changing concepts in hypertension management. J Hum Hypertens. 2017;31:763-7.

5. Weber MA, Schiffrin EL, White WB, Mann S, Lindholm LH, Kenerson JG, et al. Clinical practice guidelines for the management of hypertension in the community. A statement by the American Society of Hypertension and the International Society of Hypertension. J Clin Hypertens (Greenwich). 2014;16:14-26.

6. James PA, Oparil S, Carter BL, Cushman WC, DennisonHimmelfarb C, Handler J, et al. 2014 evidence-based guideline for the management of high blood pressure in adults: report from the panel members appointed to the Eighth Joint National Committee (JNC 8). JAMA. 2014;5:507-20.

7. Ministerio de Salud y Protección Social Colciencias. Guía de Práctica Clínica Hipertensión arterial primaria Número 18. Colombia: Departamento administrativo Colciencias; 2013 [Acceso abril de 2015]. Disponible en: http://gpc. minsalud.gov.co/Documents/Guias-PDF-Recursos/HTA/GPC Completa_HTA.pdf

8. Go AS, Bauman MA, Coleman King SM, Fonarow GC, Lawrence W, Williams KA, et al. An effective approach to high blood pressure control: a science advisory from the American Heart Association, the American College of Cardiology, and the Centers for Disease Control and Prevention. J Am Coll Cardiol. 2014;1:1230-8.

9. Redón J, Coca A, Lázaro P, Aguilar MD, Cabañas M, Gil N, et al. Factors associated with therapeutic inertia in hypertension: validation of a predictive model. J Hypertens. 2010;28:1770-7.

10. Phillips LS, Branch WT, Cook CB, Doyle JP, ElKebbi IM, Gallina DL, et al. Clinical inertia. Ann Intern Med. 2001;135:825-34.

11. Machado Duque ME, Ramírez Valencia DM, Medina Morales DA, Machado Alba JE. Effectiveness and clinical inertia in the management of hypertension in patients in Colombia. J Am Soc Hyperten. 2015;9:878-84.

12. Lebeau JP, Cadwallader JS, Aubin-Auger I, Mercier A, Pasquet T, Rusch E, et al. The concept and definition of therapeutic inertia in hypertension in primary care: a qualitative systematic review. BMC Fam Pract. 2014;2:130.

13. Benavides VA, Rosero LJ, Rendón SM, Valenzuela AM, Pérez EA, Mafla AC. Determinantes de adherencia al tratamiento antihipertensivo de adultos $\geq 35$ años de edad. Universidad y Salud. 2013;15:123-35.

14. National High Blood Pressure Education Program. The Seventh Report of the Joint National Committee on Prevention, Detection, Evaluation, and Treatment of High Blood Pressure. Bethesda (MD): National Heart, Lung, and Blood Institute (US); 2004 Aug. Disponible en: http://www.ncbi.nlm.nih.gov/books/ NBK9630/

15. Unity's Clinical Quality Improvement Committee. The Clinical Practice Guidelines for Hypertension Adult was reviewed and approved; 2014. [Acceso 21 Mar 2014]. Disponible en: https:// unityhealth.com/docs/default-source/docs/clincialguidelinesh ypertension.pdf?sfvrsn=2

16. Wu HY, Huang JW, Lin HJ, Liao WC, Peng YS, Hung KY, et al. Comparative effectiveness of renin-angiotensin system blockers 
and other antihypertensive drugs in patients with diabetes: systematic review and bayesian network meta-analysis. BMJ. 2013;347:f6008.

17. Christe V, Waeber G, Vollenweider P, Marques Vidal P. Antihypertensive drug treatment changes in the general population: the CoLaus study. BMC Pharmacol Toxicol. 2014;31:20.

18. Ueda T, Kai H, Imaizumi T. MAPPY Study Investigators. Losartan/hydrochlorothiazide combination vs. high-dose losartan in patients with morning hypertension-a prospective, randomized, open-labeled, parallel-group, multicenter trial. Hypertens Res. 2012;35:708-14.

19. Byrd JB, Bakris G, Jamerson K. The contribution of the ACCOMPLISH trial to the treatment of stage 2 hypertension. Curr Hypertens Rep. 2014;16:419.

20. Jamerson K, Weber MA, Bakris GL, et al. Benazepril plus amlodipine or hydrochlorothiazide for hypertension in high-risk patients. N Engl J Med. 2008;359:2417-28.

21. Taddei S. Combination therapy in hypertension: what are the best options according to clinical pharmacology principles and controlled clinical trial evidence? Am J Cardiovasc Drugs. 2015;15:185-94.

22. Portilla A, Torres D, Machado-Duque ME, Machado-Alba JE. Intervención para la racionalización del uso de losartán. Rev Colomb Cardiol. 2017;24:10-4.

23. Fernández Urrusuno R, Pérez Pérez P, Montero Balosa MC, González Limones S, Caraballo Camacho MO, Cuberos Fernández V. Quality prescribing of long-acting beta-agonists. Results of an intervention in Primary Care. Rev Calid Asist. 2012;27:11-8.

24. Lebeau JP, Cadwallader JS, Aubin Auger I, Mercier A, Pasquet T, Rusch E, et al. The concept and definition of therapeutic inertia in hypertension in primary care: a qualitative systematic review. BMC Fam Pract. 2014;15:130.

25. Gil Guillen V, Orozco Beltrán D, Márquez Contreras E, Durazo Arvizu R, Cooper R, Pita-Fernández S, et al. Is there a predictive profile for clinical inertia in hypertensive patients? Drugs Aging. 2011;28:981-92.

26. Feldman RD, Liu L, Wu Z, Zhang Y, Yu X, Zhang XH. Hypertension Attitude Perspectives and Needs (HAPPEN): A Real-World Survey of Physicians and Patients With Hypertension in China. J Clin Hypertens (Greenwich). 2017;19: 256-64.

27. Mu L, Mukamal KJ. Treatment Intensification for Hypertension in US Ambulatory Medical Care. J Am Heart Assoc. 2016;22, 5(10)pii:e004188.

28. Machado Alba J. ¿Inercia clínica, que tanto nos afecta? Rev Méd Risaralda. 2013;19:94-6.

29. Romero M, Arango C. Análisis de costo efectividad del uso de metoprolol succinato en el tratamiento de la hipertensión arterial y la falla cardiaca en Colombia. Rev Colomb Cardiol. 2012;19:160-8.

30. Bronsert MR, Henderson WG, Valuck R, Hosokawa P, Hammermeister K. Comparative effectiveness of antihypertensive therapeutic classes and treatment strategies in the initiation of therapy in primary care patients: a Distributed Ambulatory Research in Therapeutics Network (DARTNet) study. J Am Board Fam Med. 2013;26:529-38.

31. Gómez-Huelgas R, Martínez-Castelao A, Artola S, Górriz JL, Menéndez E. Consensus document on treatment of type 2 diabetes in patients with chronic kidney disease. Nefrologia. 2014;34:34-45.

32. McLean DL, Simpson SH, McAlister FA, Tsuyuki RT. Treatment and blood pressure control in 47,964 people with diabetes and hypertension: A systematic review of observational studies. Can J Cardiol. 2006;22:855-60.

33. López-Simarro F, Inercia terapéutica. Causas y soluciones. Hipertens Riesgo Vasc. 2012;29:28-33.

34. Branch WT, Higgins S. Inercia clínica: la dificultad de superarla. Rev Esp Cardiol. 2010;63:1399-401. 